\title{
TOPOLOGY OF SURFACES WITH CONNECTED SHADES
}

\author{
MOHAMMAD GHOMI
}

\begin{abstract}
We prove that any closed orientable surface may be smoothly embedded in Euclidean 3-space so that when it is illuminated by parallel rays from any direction the shade cast on the surface is connected.
\end{abstract}

\section{INTRODUCTION}

Let $M$ be an oriented surface smoothly immersed in Euclidean space $\mathbf{R}^{3}$, and $\nu: M \rightarrow \mathbf{S}^{2}$ be the unit normal vectorfield, or the Gauss map of $M$. Then for any unit vector $u \in \mathbf{S}^{2}$ (corresponding to the direction of light) the shade, or shadow ${ }^{1}$, cast on $M$ is defined as

$$
S_{u}:=\{p \in M \mid\langle\nu(p), u\rangle>0\},
$$

where $\langle\cdot, \cdot\rangle$ is the standard innerproduct in $\mathbf{R}^{3}$. A basic question, first considered by H. Wente in 1978 [19], is: Does connectedness of each of the shades $S_{u}$ of a closed orientable surface $M$ imply that $M$ is convex? In [5] the author showed that the answer is yes provided that either $M$ is topologically a sphere, or each of its shades is simply connected. Otherwise, it was proved that the answer is no by constructing smooth $\left(\mathcal{C}^{\infty}\right)$ embedded tori with connected shades. In this paper we extend that construction to all orientable closed surfaces:

Theorem 1.1. Every orientable closed surface admits a smooth embedding in $\mathbf{R}^{3}$ with connected shades in all directions.

Thus, surprisingly, topological complexity has in general no bearing on the number of components of a shade of a surface. In particular, the above theorem gives counterexamples of every genus to a conjecture of J. Choe [3, p. 210] which states that any immersion of a surface of topological genus $g$ in $\mathbf{R}^{3}$ should have at least one shade with $g+1$ components.

1991 Mathematics Subject Classification. Primary 53A05, 53A04; Secondary 52A15, $53 \mathrm{C} 42$.

Key words and phrases. Shadow, shade, vision number, skew loop, tantrix, constant mean curvature, gauss map, two-piece-property, tight immersion.

Supported by NSF Grant DMS-0336455, and CAREER award DMS-0332333.

Date: Summer 2002. Last typeset: September 12, 2005.

${ }^{1}$ While the author used the term "shadow" in [5], it is preferable to use "shade" as it is more suggestive, and it is consistent with the literature on computer vision. 
Note that $M$ has connected shades if and only if for every great circle $C \subset$ $\mathbf{S}^{2}, \nu^{-1}\left(\mathbf{S}^{2}-C\right)$ has exactly two components. That is, the Gauss map of $M$ satisfies a two-piece-property [2], or tightness, in the sense of Banchoff [1] or Kuiper [14]. The number of components of $\nu^{-1}\left(\mathbf{S}^{2}-C\right)$ has been called the vision number [3] with respect to a direction perpendicular to $C$, and is of interest in variational problems in geometric analysis, particularly the study of the stability of surfaces of constant mean curvature (soap films) [3, 4]. The study of shades is also of substantial interest in computer vision $[12,13]$, where "shape from shading" problems are studied extensively.

The proof of Theorem 1.1, which is presented in Section 4, follows from a pair of preliminary results, Propositions 2.1 and 3.1, proved in the next two sections. The first proposition is concerned with the existence of closed curves without any pairs of parallel tangent lines, i.e., skew loops, and is an extension of a construction first discovered by B. Segre [16], see [5, Note 6.6]. It was shown in [5] that a tubular surface about a skew loop has connected shades. Here we show that one may construct a skew loop so that the corresponding tubular surface has any desired number of pairs of points which face away from each other, as defined in Section 3. We will then prove our second proposition which states that if a surface with connected shades has a pair of points $p, q$ which face away from each other, then one may add a 'handle' to that surface and thus increase its topological genus while preserving the connectedness of each of its shades; see Figure 1.
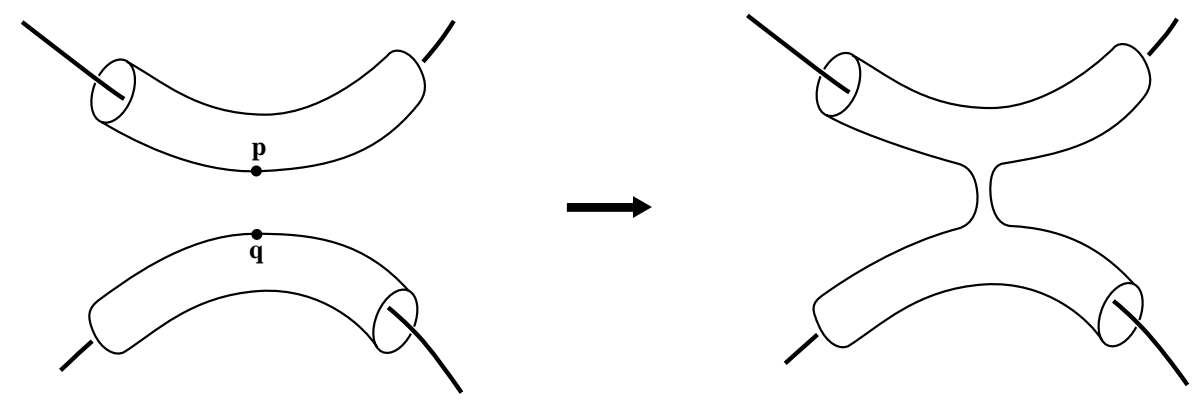

FiguRE 1

More precisely, we will delete small neighborhoods of $p$ and $q$ which are homeomorphic to disks and glue in their place a topological annulus. To this end we first deform neighborhoods of $p$ and $q$ until they coincide with pieces of spheres of the same radius, and then cut small disks from these spherical pieces. It will be shown that the resulting surface still has connected shades. Next we join the two boundary components of this surface with a surface of revolution which we call an hour glass. The hour glass has the crucial property 
that each component of each of its shades intersects its boundary. This implies that our final surface will have connected shades.

\section{Skew Loops with Prescribed Points, Tangents, AND PRINCIPAL NORMALS}

By a curve in this paper we mean a continuous mapping $\gamma: \mathbf{R} \rightarrow \mathbf{R}^{3}$. We say $\gamma$ is closed, or is a loop, if it is periodic, and write $\gamma: \mathbf{R} / \ell \rightarrow \mathbf{R}^{3}$, where $\ell$ denotes the period. Further, $\gamma$ is simple if $\gamma(t) \neq \gamma(s)$, whenever $0<|t-s|<\ell$. A regular curve is a $\mathcal{C}^{1}$ mapping $\gamma: \mathbf{R} \rightarrow \mathbf{R}^{3}$ with nonvanishing speed, i.e., $\left\|\gamma^{\prime}\right\| \neq 0$. The tangential indicatrix or tantrix of a regular curve $\gamma$ is given by $T:=\gamma^{\prime} /\left\|\gamma^{\prime}\right\|$. If $T(t) \neq \pm T(s)$, whenever $t \not \equiv s(\bmod \ell)$, we say that $\gamma$ is skew. When $\gamma$ is $\mathcal{C}^{2}$ and regular, its curvature is defined as $\kappa:=\left\|T^{\prime}\right\| /\left\|\gamma^{\prime}\right\|$, and, if $\kappa$ does not vanish, the principal normal of $\gamma$ is given by $N:=T^{\prime} / \kappa$. By smooth in this paper we always mean $\mathcal{C}^{\infty}$. The main aim of this section is to show:

Proposition 2.1. Let $\left(p_{i}, T_{i}, N_{i}\right) \in \mathbf{R}^{3} \times \mathbf{S}^{2} \times \mathbf{S}^{2}, i=1, \ldots, n$. Suppose that $T_{i} \neq \pm T_{j}$ whenever $i \neq j$, and $\left\langle N_{i}, T_{i}\right\rangle=0$. Then there exists a smooth skew loop $\gamma: \mathbf{R} / n \rightarrow \mathbf{R}^{3}$ with nonvanishing curvature such that

$$
\gamma(i)=p_{i}, \quad T(i)=T_{i}, \quad \text { and } \quad N(i)=N_{i},
$$

where $T$ and $N$ are the tantrix and the principal normal of $\gamma$ respectively. Furthermore, if $p_{i} \neq p_{j}$ whenever $i \neq j$, then we may require that $\gamma$ be simple.

As we mentioned earlier, the above proposition may be regarded as a generalization of an earlier work of B. Segre [16] who first proved the existence of skew loops (without prescribing specific points or frames). See [11] for some historical notes on skew loops, and see [5] for an explicit example. More recent developments on the theory of skew submanifolds may be found in $[9,8,10,18,17]$. To prove the above proposition we need the following lemmas:

Lemma 2.2. Let $\left(T_{i}, N_{i}\right) \in \mathbf{S}^{2} \times \mathbf{S}^{2}, i=1, \ldots, n$. Suppose that $T_{i} \neq \pm T_{j}$ whenever $i \neq j$, and $\left\langle N_{i}, T_{i}\right\rangle=0$. Then there exists a smooth simple loop $T: \mathbf{R} / n \rightarrow \mathbf{S}^{2}$ such that

$$
T(i)=T_{i}, \quad T^{\prime}(i) \| N_{i}, \quad \text { and } \quad T(\mathbf{R}) \cap-T(\mathbf{R})=\emptyset .
$$

Proof. It suffices to show that there exists a simply connected open subset $U \subset \mathbf{S}^{2}$ such that $T_{i} \in U$ and $U \cap-U=\emptyset$; for one may then easily construct, by induction, a simple closed regular curve $T: \mathbf{R} / n \rightarrow U$ with $T(i)=T_{i}$ and $T^{\prime}(i) \| N_{i}$. Any such curve would automatically satisfy $T(\mathbf{R}) \cap-T(\mathbf{R})=\emptyset$, and thus would be the desired object.

To construct $U$ we first note that, when $i \neq j$, every pair of points $T_{i}, T_{j}$ determine a unique great circle $C_{i j}$ passing through them, since $T_{i} \neq \pm T_{j}$ when $i \neq j$. Let $C:=\left\{C_{i j}\right\}$ be the collection of all such circles, and pick a 
point $o \in \mathbf{S}^{2}-C$. Then $o \neq-T_{i}$. So every $T_{i}$ may be joined to $o$ by a unique distance minimizing geodesic segment $\Gamma_{i}$. Further, since $o \notin C$, no pairs of geodesic segments $\Gamma_{i}$ and $\Gamma_{j}$ can be parts of the same great circle when $i \neq j$. This implies that $-\Gamma_{i} \cap \Gamma_{j}=\emptyset$ for all $i \neq j$. Further, since by assumption $\Gamma_{i}$ is distance minimizing, i.e., its length is less than half of a great circle; we have $\Gamma_{i} \cap-\Gamma_{i}=\emptyset$. So if $\Gamma:=\cup_{i} \Gamma_{i}$, then $\Gamma \cap-\Gamma=\emptyset$. In particular $\delta:=\operatorname{dist}_{\mathbf{S}^{2}}(\Gamma,-\Gamma)>0$. Now let $0<\epsilon<\delta / 2$, and, for any $p \in \mathbf{S}^{2}$, let $U_{\epsilon}(p)$ denote the set of points in $\mathbf{S}^{2}$ whose (spherical) distance from $p$ is less than $\epsilon$. Then $U:=\cup_{p \in \Gamma} U_{\epsilon}(p)$ has the required properties.

For any $X \subset \mathbf{R}^{3}$, the cone of $X$ is defined as

$$
\text { cone } X:=\{\lambda x \mid x \in X \text {, and } \lambda \geq 0\},
$$

and the convex hull of $X$ is the intersection of all convex sets containing $X$. By the convex cone of $X$ we mean the convex hull of the cone of $X$.

Lemma 2.3. Let $T:[a, b] \rightarrow \mathbf{S}^{2}$ be any continuous map. Then for any point $p$ in the interior of the convex cone of $T([a, b])$, there exists a smooth positive function $\varphi:[a, b] \rightarrow \mathbf{R}$ such that

$$
\int_{a}^{b} \varphi(t) T(t) d t=p .
$$

Further, there exists $\bar{\delta}>0$ such that for any $0<\delta \leq \bar{\delta}$ we may set $\varphi$ equal to $\delta$ on an open neighborhood of the end points of $[a, b]$.

Proof. By a theorem of Steinitz [15, p. 15], any interior point of the convex hull of $X \subset \mathbf{R}^{3}$ lies in the interior of the convex hull of 6 (or fewer) points of $X$. So, since $p \in$ int conv cone $T([a, b])=$ int conv cone $T((a, b))$, there exist $t_{i} \in(a, b)$, and $c_{i}>0, i=1, \ldots, k$, where $4 \leq k \leq 6$, such that

$$
p \in \operatorname{int} \operatorname{conv}\left\{c_{1} T\left(t_{1}\right), \ldots, c_{k} T\left(t_{k}\right)\right\} \text {. }
$$

Let $\varphi_{i}^{\epsilon}:[a, b] \rightarrow \mathbf{R}$ be a smooth nonnegative function with $\int_{a}^{b} \varphi_{i}^{\epsilon}(t) d t=1$, and support in an $\epsilon$-neighborhood of $t_{i}$. Note that since $t_{i} \in(a, b)$, we may assume that

$$
0<\epsilon<\min _{i}\left\{\left|t_{i}-a\right|,\left|t_{i}-b\right|\right\}
$$

which implies that $\varphi_{i}^{\epsilon}$ vanishes near $a$ and $b$. Let

$$
T_{i}^{\epsilon}:=\int_{a}^{b}\left(\varphi_{i}^{\epsilon}(t)+\epsilon\right) c_{i} T(t) d t .
$$

Then $\lim _{\epsilon \rightarrow 0} T_{i}^{\epsilon}=c_{i} T\left(t_{i}\right)$. In particular, by (2.1), we may choose $\epsilon$ small enough so that

$$
p \in \operatorname{int} \operatorname{conv}\left\{T_{1}^{\epsilon}, \ldots, T_{k}^{\epsilon}\right\}
$$


Then, by a standard result from classical convexity theory [15, Thm. 1.1.13], there are constants $\lambda_{i}^{\epsilon}>0$, with $\sum_{i=1}^{k} \lambda_{i}^{\epsilon}=1$, such that

$$
p=\sum_{i=1}^{n} \lambda_{i}^{\epsilon} T_{i}^{\epsilon}
$$

Now set

$$
\varphi^{\epsilon}:=\sum_{i=1}^{k} \lambda_{i}^{\epsilon}\left(\varphi_{i}^{\epsilon}+\epsilon\right) c_{i}
$$

Then $\varphi^{\epsilon}>0$, because $c_{i}, \lambda_{i}^{\epsilon}, \epsilon>0$. Further

$$
\int_{a}^{b} \varphi^{\epsilon}(t) T(t) d t=p
$$

Also note that, whenever (2.2) is satisfied, $\varphi^{\epsilon}$ is equal to

$$
\bar{\delta}(\epsilon):=\epsilon \sum_{i=1}^{k} \lambda_{i}^{\epsilon} c_{i}
$$

near $a$ and $b$, because $\varphi_{i}^{\epsilon}$ vanishes near $a$ and $b$.

Let $\epsilon$ be small enough so that (2.2) and (2.3) are satisfied, and set

$$
\bar{T}_{i}^{\epsilon}:=\int_{a}^{b} \varphi_{i}^{\epsilon}(t) c_{i} T(t) d t
$$

Then $\lim _{\epsilon \rightarrow 0} \bar{T}_{i}^{\epsilon}=c_{i} T\left(t_{i}\right)$, and just as we had argued above, we may choose $\epsilon$ small enough so that, in addition to (2.2) and (2.3), the following condition is also satisfied:

$$
p \in \operatorname{int} \operatorname{conv}\left\{\bar{T}_{1}^{\epsilon}, \ldots, \bar{T}_{k}^{\epsilon}\right\} .
$$

Then, again by [15, Thm. 1.1.13], there are constants $\bar{\lambda}_{i}^{\epsilon}>0$, with $\sum_{i=1}^{k} \bar{\lambda}_{i}^{\epsilon}=$ 1 , such that

$$
p=\sum_{i=1}^{k} \bar{\lambda}_{i}^{\epsilon} \bar{T}_{i}^{\epsilon}
$$

Now if we set

$$
\bar{\varphi}^{\epsilon}:=\sum_{i=1}^{k} \bar{\lambda}_{i}^{\epsilon} \varphi_{i}^{\epsilon} c_{i},
$$

then,

$$
\int_{a}^{b} \bar{\varphi}^{\epsilon}(t) T(t) d t=p .
$$


Finally, for fixed $\epsilon$ satisfying (2.2), (2.3), (2.5) and any $0<\delta \leq \bar{\delta}(\epsilon)$, set

$$
\varphi:=\frac{\delta}{\bar{\delta}(\epsilon)} \varphi^{\epsilon}+\left(1-\frac{\delta}{\bar{\delta}(\epsilon)}\right) \bar{\varphi}^{\epsilon} .
$$

Then $\int_{a}^{b} \varphi(t) T(t) d t=p$ by (2.4) and (2.6). Also recall that $\varphi_{\epsilon}>0$; thus $\varphi>0$. Finally note that $\varphi=\delta$ near $a$ and $b$, because in that region $\bar{\varphi}_{i}^{\epsilon}$ and consequently $\bar{\varphi}^{\epsilon}$ vanish by $(2.2)$ and $\varphi^{\epsilon}=\bar{\delta}(\epsilon)$.

Finally we need to recall the following basic fact, which will be used to prove the last assertion in the statement of Proposition 2.1.

Lemma 2.4 ([11]). $\mathcal{C}^{2}$ skew loops with nonvanishing curvature form an open subset in the space of all $\mathcal{C}^{2}$ closed curves $\gamma: \mathbf{R} / \ell \rightarrow \mathbf{R}^{3}$, with respect to the $\mathcal{C}^{2}$ topology.

Now we are ready to prove the main result of this section:

Proof of Proposition 2.1. Let $v_{j} \in \mathbf{S}^{2}, j=1, \ldots, 4$, be four points which contain the origin of $\mathbf{R}^{3}$ in the interior of their convex hull,

$$
o \in \operatorname{int} \operatorname{conv}\left\{v_{1}, v_{2}, v_{3}, v_{4}\right\} .
$$

In particular, $v_{i} \neq \pm v_{j}$, whenever $i \neq j$. Further suppose that $v_{j} \neq \pm T_{i}$. Then there exist open neighborhoods $U_{j}$ of $v_{j}$ in $\mathbf{S}^{2}$ such that $U_{i} \cap \pm U_{j}=\emptyset$ whenever $i \neq j, \pm T_{i} \notin U_{j}$ for all $i$ and $j$, and if $y_{j} \in U_{j}$ then the interior of the convex hull of $\left\{y_{1}, \ldots, y_{4}\right\}$ contains the origin. Now let $v_{i j} \in U_{j}, i=1, \ldots n$, be a sequence of distinct points, and $\bar{T}_{k} \in \mathbf{S}^{2}, k=1, \ldots, 5 n$ be the sequence whose elements are

$$
T_{1}, v_{11}, v_{12}, v_{13}, v_{14}, T_{2}, v_{21}, v_{22}, v_{23}, v_{24}, \ldots, T_{n}, v_{n 1}, v_{n 2}, v_{n 3}, v_{n 4} .
$$

Then $\bar{T}_{i} \neq \pm \bar{T}_{j}$, whenever $i \neq j$. Let $\bar{N}_{k} \in T_{\bar{T}_{k}} \mathbf{S}^{2}, k=1, \ldots, 5 n$, be any sequence with $\bar{N}_{5 i-4}:=N_{i}, i=1, \ldots, n$. Applying Lemma 2.2 to $\bar{T}_{k}$ and $\bar{N}_{k}$, we obtain, after a reparametrization, a smooth simple closed curve $T: \mathbf{R} / n \rightarrow$ $\mathbf{S}^{2}$ such that, for $i=1, \ldots, n$,

$$
T(i)=T_{i}, \quad T^{\prime}(i) \| N_{i} \quad \text { and } \quad\left\{v_{i 1}, v_{i 2}, v_{i 3}, v_{i 4}\right\} \subset T([i, i+1]) .
$$

The last condition implies that the origin lies in the interior of the convex hull of $T([i, i+1])$. Thus conv cone $T([i, i+1])=\mathbf{R}^{3}$, and, in particular,

$$
\left(p_{i+1}-p_{i}\right) \in \text { int conv cone } T([i, i+1]) \text {. }
$$

So it follows from Lemma 2.3 that fo each $i$ there exists a smooth positive function $\varphi_{i}:[i, i+1] \rightarrow \mathbf{R}$ such that

$$
\int_{i}^{i+1} \varphi_{i}(t) T(t) d t=p_{i+1}-p_{i},
$$


where we set $p_{n+1}:=p_{1}$. Further, on an open neighborhood of the end points of $[i, i+1]$ we may set $\varphi_{i}$ equal to any positive constant smaller than or equal to some positive constant $\bar{\delta}_{i}$. In particular we may assume that $\varphi_{i}$ is equal to $\bar{\delta}:=\min _{i} \delta_{i}$ near $i$ and $i+1$. Then, $\varphi: \mathbf{R} / n \rightarrow \mathbf{R}$ defined by

$$
\left.\varphi\right|_{[i, i+1]}:=\varphi_{i}
$$

is a smooth function. Now define $\gamma: \mathbf{R} / n \rightarrow \mathbf{R}$ by

$$
\gamma(t):=p_{1}+\int_{1}^{t} \varphi(s) T(s) d s .
$$

Then $\gamma(i)=p_{i}$, and $\gamma^{\prime}(i) \| T_{i}, i=1, \ldots, n$. Further $\gamma^{\prime} /\left\|\gamma^{\prime}\right\|=T$. So $T$ is the tantrix of $\gamma$. Thus, since by Lemma $2.2 T$ is regular, i.e., $\left\|T^{\prime}\right\| \neq 0$, it follows that the curvature $\kappa$ of $\gamma$ does not vanish. Furthermore, by Lemma 2.2, $T$ is one-to-one on $\mathbf{R} / n$ and $T(\mathbf{R}) \cap-T(\mathbf{R})=\emptyset$. Thus $T$ is skew. Finally recall that $N:=T^{\prime} /\left\|T^{\prime}\right\|$. So, since $T^{\prime}(i) \| N_{i}$, it follows that $N(i)=N_{i}$.

To prove the last assertion of the proposition, it only remains to note that, using the Wierstrauss approximation theorem followed by gluings, we may construct a smooth closed curve $\widetilde{\gamma}: \mathbf{R} / n \rightarrow \mathbf{R}^{3}$ which coincides with $\gamma$ in a neighborhood of each integer and is $\mathcal{C}^{2}$-close to $\gamma$. In particular, if $p_{i} \neq p_{j}$, for all $i \neq j$, then we may assume that $\widetilde{\gamma}$ is simple (since embedding are dense in the space of immersions of an $n$-manifold into a $2 n+1$-manifold). Further, Lemma 2.4 ensures that $\widetilde{\gamma}$ is skew.

\section{Adding Handles to Surfaces with Connected Shades}

Let $M \subset \mathbf{R}^{3}$ be a $\mathcal{C}^{2}$ immersed surface and $p, q \in M$. We say that $p$ and $q$ face away from each other provided that (i) the line segment $p q$ is orthogonal to $M$ at $p$ and $q$, (ii) the Gaussian curvature of $M$ is positive at $p$ and $q$, and (iii) if $H$ is the mean curvature vector of $M$, then $H(p) \| p-q$ and $H(q) \| q-p$; see Figure 2. The main result of this section is:

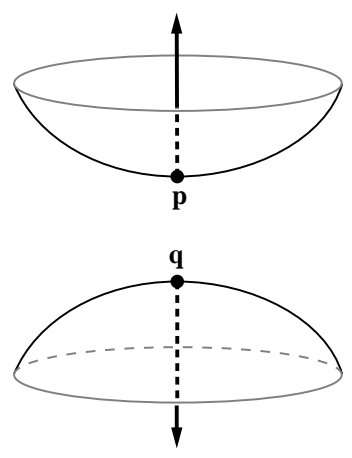

FiguRE 2 
Proposition 3.1. Let $M \subset \mathbf{R}^{3}$ be a smooth closed embedded surface with connected shades. Suppose $M$ has a pair of points $p, q$ which face away from each other. Then there exists a smooth closed embedded surface $\widetilde{M} \subset \mathbf{R}^{3}$ with connected shades, and

$$
g(\widetilde{M})=g(M)+1
$$

Further, for any open neighborhood $U$ of $\{p, q\}$ in $M$ and $\epsilon>0$, we may require that $\widetilde{M} \supset(M-U)$, and $\widetilde{M}-M$ lie within an $\epsilon$ neighborhood of the line segment $p q$.

The proof of the above result rests on the following lemmas, the first of which is concerned with a simple topological fact:

Lemma 3.2. Let $M$ be a closed surface which is obtained by gluing a pair of surfaces $M_{1}$ and $M_{2}$ along their boundary. Suppose there exists an open set $U \subset M$ such that $U \cap M_{1}$ is connected and each component of $U \cap M_{2}$ intersects $\partial M_{2}$. Then $U$ is connected.

Proof. If $U \cap M_{2}=\emptyset$ we are done. Otherwise, let $V$ be a nonempty component of $U \cap M_{2}$. Then, by assumption, $V$ intersects $\partial M_{2}$. Let $W$ be the component of $U$ which contains $V$. Then $W \cap \partial M_{2} \neq \emptyset$. So, since $W$ is open in $M$, it must contain a point of $M_{1}$. In particular, $W$ intersects $U \cap M_{1}$. Therefore, since $U \cap M_{1}$ is connected, $W$ must contain $U \cap M_{1}$. Thus every component of $U \cap M_{2}$ lies in the (unique) component of $U$ which contains $U \cap M_{1}$. So $U$ is connected.

The next four lemmas are concerned with local deformations of a surface and their effects on the connectedness of shades of that surface. We say that a $\mathcal{C}^{2}$ hypersurface $M^{n}$ immersed in $\mathbf{R}^{n+1}$ is strictly convex, if (i) $M$ has positive Gauss curvature, (ii) through every point $p$ of $M$ there passes a plane $\Pi$ such that $\Pi \cap M=\{p\}$, and (iii) $M$ lies on one side of $\Pi$. Note that, in our terminology, a strictly convex surface may not be connected.

Lemma 3.3 ([7]). Every smooth compact strictly convex hypersurface $M \subset$ $\mathbf{R}^{n+1}$ may be extended to a smooth closed embedded hypersurface $O$ of positive curvature. Further, we may require that $O$ lie within an arbitrary small distance of the convex hull of $M$.

The above lemma yields:

Lemma 3.4. Let $M \subset \mathbf{R}^{3}$ be a smooth embedded surface, and $p \in M$ be an interior point with positive Gauss curvature. Then for any open neighborhood $U$ of $p$ in $M$ which has positive Gauss curvature there exists a smooth embedded surface $M^{\prime}$ homeomorphic to $M$, and arbitrarily $C^{1}$-close to $M$, such that $M^{\prime} \supset$ $M-U, M^{\prime}-(M-U)$ has positive Gauss curvature, $p \in M^{\prime}, T_{p} M^{\prime}=T_{p} M$, and an open neighborhood of $p$ in $M^{\prime}$ lies on a sphere of radius $r$, for any given $r>0$. 
Proof. First note that, without loss of generality, we may replace $U$ by any open neighborhood of $p$ contained in $U$. In particular, we may assume that $\bar{U}$ is homeomorphic to a disk, is strictly convex, and is a graph over $T_{p} M$. Further, for definiteness we may assume, after a rigid motion, that $T_{p} M$ coincides with the $x y$ plane and $\bar{U}$ lies above $x y$-plane.

Now let $A \subset \bar{U}$ be an open neighborhood of $\partial U$, such that $p \notin \bar{A}$, and let $S$ be a sphere of radius of $r$ which is tangent to $M$ at $p$ and lies above the $x y$-plane; see Figure 3 . Note that, since $\bar{U}$ is strictly convex, every tangent
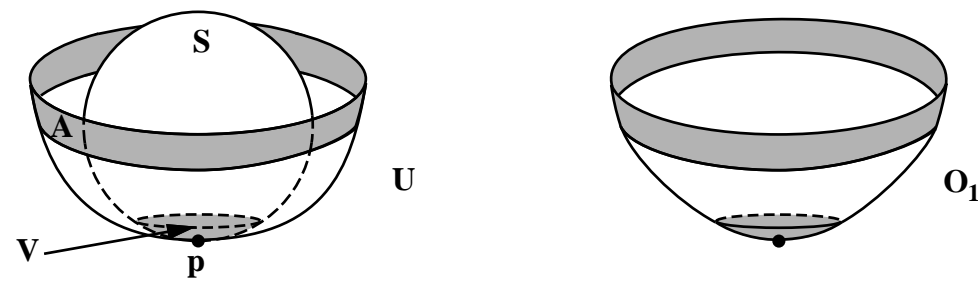

\section{FiguRE 3}

plane of $\bar{A}$ is disjoint from $p$. Further, $T_{p} S=T_{p} U$ is disjoint from $\bar{A}$. So, since $\bar{A}$ is compact, it follows that there exists an open neighborhood $V$ of $p$ in $S$ such that $\bar{V} \cup \bar{A}$ is strictly convex. Consequently, by Lemma 3.3, there exists a smooth closed surface of positive Gauss curvature $O \supset \bar{A} \cup \bar{V}$. By Jordan's curve theorem, $O-\partial U$ consists of precisely two components. Let $O_{1}$ be the component of $O-\partial U$ which contains $p$, and $M^{\prime}$ be the closed surface obtained from gluing $M-U$ to $O_{1}$ along their common boundary.

Note that $A-\partial U \subset O_{1}$. Thus $M^{\prime}$ is smooth. Further, by Schoenflies theorem, $O_{1}$ is homeomorphic to a disk. Thus, since $U$ is also homeomorphic to a disk, and $M^{\prime}-O_{1}=M-U$, it follows that $M^{\prime}$ is homeomorphic to $M$. Next note that, $V \subset O_{1} \subset M^{\prime}$. So $T_{p} M=T_{p} M^{\prime}$. Finally note that, since $T_{p} M=T_{p} M^{\prime}$ and $O_{1}$ is convex, we may choose $U$ small enough so that $M^{\prime}$ is as close to $M$ as desired in the sense of $C^{1}$-topology. Thus, since $M$ is compact and embedded, we can make sure that $M^{\prime}$ is embedded as well.

The proof of the next lemma employs the following simple but useful observation, which will be invoked a number of times in the remainder of this paper. For every $u \in \mathbf{S}^{2}$ let

$$
H_{u}:=\left\{p \in \mathbf{S}^{2} \mid\langle p, u\rangle>0\right\}
$$

be the (open) hemisphere of $\mathbf{S}^{2}$ centered at $u$. Then if $M$ is any oriented surface immersed in $\mathbf{R}^{3}$ and $\nu: M \rightarrow \mathbf{S}^{2}$ is its Gauss map, we have

$$
S_{u}=\nu^{-1}\left(H_{u}\right)
$$


for all $u \in \mathbf{S}^{2}$. We say that a set $X \subset \mathbf{S}^{2}$ is geodesically convex if it lies in an open hemisphere and every pairs of points of $X$ may be joined by a geodesic segment which lies in $X$.

Lemma 3.5. Let $M \subset \mathbf{R}^{3}$ be a smooth oriented closed surface with connected shades, and $U \subset M$ be an open set such that $\left.\nu\right|_{\bar{U}}$ is a diffeomorphism into a geodesically convex set. Then $M-U$ has connected shades.

Proof. Let $\widetilde{S}_{u}$ be a shade of $\widetilde{M}:=M-U$. Then $\widetilde{S}_{u}:=S_{u}-U$. In particular, $\widetilde{S}_{u}$ is connected if $U \cap S_{u}=\emptyset$. So we may assume that $U \cap S_{u} \neq \emptyset$; further, we may assume $S_{u} \not \subset U$, for otherwise $\widetilde{S}_{u}$ would be empty. These two assumptions yield that $S_{u} \cap \partial U \neq \emptyset$. Since $S_{u}$ is connected, this implies that every component of $\widetilde{S}_{u}$ must intersect $\partial U$. So, to show that $\widetilde{S}_{u}$ is connected, it suffices to check that $\widetilde{S}_{u} \cap \partial U$ is connected. But $\widetilde{S}_{u} \cap \partial U=S_{u} \cap \partial U$, and $S_{u} \cap \partial U=$ $\nu^{-1}\left(H_{u} \cap \nu(\partial U)\right)$. Thus, since by assumption $\nu^{-1}: \nu(\bar{U}) \rightarrow U$ is continuous, we just need to check that $H_{u} \cap \nu(\partial U)$ is connected. This follows from the assumption that $\nu(\bar{U})$ is geodesically convex. To see this assume that $\nu(\bar{U})$ lies in $H_{(0,0,1)}$, and take a stereographic projection from the center of $\mathbf{S}^{2}$ to obtain a homeomorphism $\pi: H_{(0,0,1)} \rightarrow T_{(0,0,1)} \mathbf{S}^{2} \simeq \mathbf{R}^{2}$. Then $\pi(\nu(\bar{U}))$ is a convex planar set, and $\pi\left(H_{(0,0,1)} \cap H_{u}\right)$ is an open half-space. Thus, by basic convexity theory, $\pi\left(\nu(\partial U) \cap H_{u}\right)=\pi(\nu(\partial U)) \cap \pi\left(H_{(0,0,1)} \cap H_{u}\right)$ is connected, which yields that $\nu(\partial U) \cap H_{u}$ is connected.

Lemma 3.6. Let $M \subset \mathbf{R}^{3}$ be a smooth oriented closed surface with connected shades, and $U \subset M$ be an open set such that $\bar{U}$ has positive Gauss curvature and $\left.\nu\right|_{\bar{U}}$ is a diffeomorphism into a geodesically convex set. If $M^{\prime}$ is any closed smooth surface such that $M^{\prime} \supset M-U$ and $M^{\prime}-(M-U)$ has positive Gauss curvature, then $M^{\prime}$ has connected shades.

Proof. Let $\nu^{\prime}: M^{\prime} \rightarrow \mathbf{S}^{2}$ be the Gauss map of $M^{\prime}$, and $U^{\prime}:=M^{\prime}-(M-U)$. Note that $\partial U^{\prime}=\partial U$, which is homeomorphic to a circle, since $\left.\nu\right|_{\bar{U}}$ is a diffeomorphism into a geodesically convex set. So $\bar{U}^{\prime}$ is a topological manifold with boundary. Next note that, since $U^{\prime}$ has positive Gauss curvature by assumption, and $\partial U^{\prime}=\partial U \subset \bar{U}$, it follows that $\overline{U^{\prime}}$ has positive Gauss curvature. So, by the inverse function theorem, $\nu^{\prime}$ is locally one-to-one on $\bar{U}^{\prime}$. Further $\nu^{\prime}$ is one-to-one on $\partial U^{\prime}$, since $\left.\nu^{\prime}\right|_{\partial U^{\prime}}=\left.\nu\right|_{\partial U}$. So it follows that $\nu^{\prime}$ in one-toone on $\bar{U}^{\prime}$; see [6] where it is proved that any locally one-to-one map from a compact $n$-manifold, $n \geq 2$, into a sphere of the same dimension is one-to-one everywhere, if it is one-to-one on the boundary of the manifold. Consequently, since $\bar{U}^{\prime}$ is compact and has nonvanishing Gauss curvature, $\nu^{\prime}: \bar{U}^{\prime} \rightarrow \nu^{\prime}\left(\bar{U}^{\prime}\right)$ is a diffeomorphism. Now, since $\left.\nu^{\prime}\right|_{\partial U^{\prime}}=\left.\nu\right|_{\partial U}$, it follows that $\nu^{\prime}\left(U^{\prime}\right)=\nu(U)$. In particular, $\nu^{\prime}\left(U^{\prime}\right)$ is geodesically convex. 
Let $S_{u}^{\prime}:=\left(\nu^{\prime}\right)^{-1}\left(H_{u}\right)$ be a shade of $M^{\prime}$. Note that if $S_{u}^{\prime} \subset U^{\prime}$, then $H_{u} \subset$ $\nu\left(U^{\prime}\right)$, which yields that $\nu\left(U^{\prime}\right)=H_{u}$; because, by definition, a geodesically convex set lies in an open hemisphere. But if $\nu\left(U^{\prime}\right)=H_{u}$, then $S_{u}^{\prime}=U^{\prime}$ which is connected, since $U^{\prime}$ is diffeomorphic to a geodesically convex set. Now suppose that $S_{u}^{\prime} \not \subset U^{\prime}$. Since $\nu^{\prime}\left(U^{\prime}\right)$ and $H_{u}$ are geodesically convex, $H_{u} \cap \nu^{\prime}\left(U^{\prime}\right)$ is geodesically convex. In particular $H_{u} \cap \nu^{\prime}\left(U^{\prime}\right)$ is connected, which yields that $S_{u}^{\prime} \cap U^{\prime}$ is connected. Further, if $S_{u}^{\prime} \cap U^{\prime} \neq \emptyset$, then $S_{u}^{\prime}$ intersects $\partial U^{\prime}$, because $S_{u}^{\prime} \not \subset U^{\prime}$. So to prove that $S_{u}^{\prime}$ is connected, it is now enough to show that $S_{u}^{\prime} \cap\left(M-U^{\prime}\right)$ is connected. To see this note that, since $M-U^{\prime}=M-U$, we have $S_{u}^{\prime} \cap\left(M-U^{\prime}\right)=S_{u} \cap(M-U)$, where $S_{u}=\nu^{-1}\left(H_{u}\right)$. So it remains to check that $S_{u} \cap(M-U)$ is connected, which is indeed the case, since, by Lemma 3.5, $M-U$ has connected shades.

By an hour glass $\mathcal{H} \subset \mathbf{R}^{3}$ we mean a smooth embedded surface of revolution, homeomorphic to an annulus, whose profile curve, when viewed as the graph of a positive function $f:[a, b] \rightarrow \mathbf{R}$, satisfies the following properties:

(1) $f^{\prime}(a)<0$ and $f^{\prime}(b)>0$,

(2) $f^{\prime \prime}<0$ on $\left[a, x_{1}\right) \cup\left(x_{2}, b\right]$ and $f^{\prime \prime}>0$ on $\left(x_{1}, x_{2}\right)$, for some $x_{1}, x_{2} \in(a, b)$; see Figure 4.

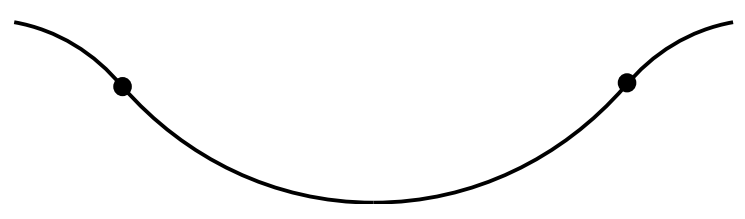

FIGURE 4

In other words, $\mathcal{H} \subset \mathbf{R}^{3}$ is an hour glass if after a rigid motion it may be parametrized by

$$
[a, b] \times \mathbf{R} \ni(z, \theta) \longmapsto(f(z) \sin (\theta), f(z) \cos (\theta), z) \in \mathbf{R}^{3},
$$

where $f:[a, b] \rightarrow \mathbf{R}$ is a smooth positive function which satisfies the properties enumerated above. The main property of an hour glass, as far as the illuminations are concerned, is stated in the next result:

Lemma 3.7. If $\mathcal{H} \subset \mathbf{R}^{3}$ is an hour glass, then every component of each shade of $\mathcal{H}$ intersects $\partial \mathcal{H}$.

Proof. By definition there are precisely two inflection points in the profile curve of $\mathcal{H}$. These points generate a pair of meridians which divide $\mathcal{H}$ into three regions. Supposing, after a rigid motion, that $\mathcal{H}$ is positioned vertically, i.e., its axis of rotation is parallel to the $z$-axis, we let $R_{1}, R_{2}$ and $R_{3}$ be the top, middle, and bottom regions respectively; see Figure 5 

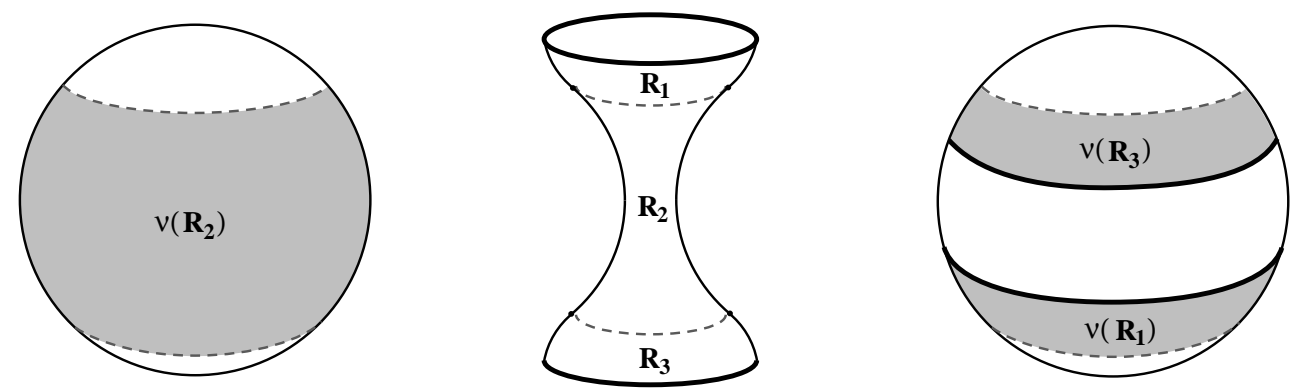

FiguRe 5

Let $\nu: \mathcal{H} \rightarrow \mathbf{S}^{2}$ be the outward unit normal of $\mathcal{H}$, and note that $\nu$ embeds each $R_{i}$ into an annular region of $\mathbf{S}^{2}$ bounded by a pair of horizontal circles or meridians. In particular, the intersection of $\nu\left(R_{i}\right)$ with any hemisphere $H_{u}$ of $\mathbf{S}^{2}$ is connected. So, by (3.1), the intersection of any shade $S_{u}$ of $\mathcal{H}$ with $R_{i}$ must be connected.

Now note that $\nu\left(R_{2}\right)$ contains the equator of $\mathbf{S}^{2}$ in its interior. Thus every great circle of $\mathbf{S}^{2}$ must intersect the interior of $\nu\left(R_{2}\right)$ (because no two great circles of $\mathbf{S}^{2}$ are disjoint). Since $\partial H_{u}$ is a great circle, it follows that $H_{u}$ intersects $\nu\left(R_{2}\right)$. But $\nu\left(R_{2}\right) \not \subset H_{u}$, because $\nu\left(R_{2}\right)$ contains a great circle and $H_{u}$ is an open hemisphere. So $H_{u}$ must intersect $\partial \nu\left(R_{2}\right)=\nu\left(\partial R_{2}\right)$. This yields that $S_{u}$ intersects $\partial R_{2}$. But, as we argued above, $S_{u} \cap R_{2}$ is connected. Thus no component $S_{u}^{1}$ of $S_{u}$ may be trapped in the interior of $\partial R_{2}$. Since $\partial R_{2} \subset\left(\partial R_{1} \cup \partial R_{3}\right)$, it follows that $S_{u}^{1}$ must intersect $R_{1}$ or $R_{3}$.

Suppose that $S_{u}^{1}$ intersects $R_{1}$. Then $H_{u}$ intersects $\nu\left(R_{1}\right)$. If $\nu\left(R_{1}\right) \subset H_{u}$, then it follows that $S_{u}^{1}$ intersects $\partial \mathcal{H}$, because a boundary component of $\nu\left(R_{1}\right)$ is the image of a boundary component of $\partial \mathcal{H}$ under $\nu$. So if $\nu\left(R_{1}\right) \subset H_{u}$ we are done. If $\nu\left(R_{1}\right) \not \subset H_{u}$, then, since $H_{u}$ intersects $\nu\left(R_{1}\right)$, it follows that $\partial H_{u}$ must intersect $\nu\left(R_{1}\right)$. But $\nu\left(R_{1}\right)$ is contained in the bottom hemisphere of $\mathbf{S}^{2}$, and $\partial H_{u}$ is a great circle. Thus $\partial H_{u}$ must intersect the upper boundary of $\nu\left(R_{1}\right)$ (otherwise, by the Jordan curve theorem, $\partial H_{u}$ would have to be contained entirely in the interior of the bottom hemisphere of $\mathbf{S}^{2}$, which would be a contradiction). But the upper boundary of $\nu\left(R_{1}\right)$ is the image of the top boundary component of $\mathcal{H}$ under $\nu$. So, since $S_{u} \cap R_{1}$ is connected, we conclude that $S_{u}^{1}$ must intersect $\partial \mathcal{H}$.

Similarly, one can show that if $S_{u}^{1}$ intersects $R_{3}$, then $S_{u}^{1}$ must intersect $\partial \mathcal{H}$, which will complete the proof.

Lemma 3.8. Let $S_{1}, S_{2} \subset \mathbf{R}^{3}$ be a pair of spheres of radius $r$, and $p_{1} \in S_{1}$, $p_{2} \in S_{2}$ be a pair of points which face away from each other. Then, for every $\epsilon>0$, there exists an hour glass $\mathcal{H}$ such that an open neighborhood of $\partial \mathcal{H}$ lies on $S_{1} \cup S_{2}$ and $\mathcal{H}$ lies within an $\epsilon$ neighborhood of the line segment $p q$. 
Proof. After a rigid motion we may assume that $p_{1}=(-a, 0,0)$ and $p_{2}=$ $(a, 0,0)$. Since, by assumption, $p_{1} p_{2}$ is orthogonal to $S_{1}$ and $S_{2}$, it follows then that $S_{1}$ and $S_{2}$ are centered at $(-a-r, 0,0)$ and $(a+r, 0,0)$ respectively. Thus the intersection of $S_{1}$ and $S_{2}$ with the $x y$-plane consists of a pair of circles of radius of $r$ centered at $(-a-r, 0)$ and $(a+r, 0)$ respectively. It suffices to show that, for every $0<\delta<r$, there exists a smooth positive function $f:[-a-\delta, a+\delta] \rightarrow \mathbf{R}$ with precisely two inflection points such that the graph of $f$ coincides with the given circles near the end points of $[-a-\delta, a+\delta]$. Revolving the graph of $f$ around the $x$-axis then yields an hour glass $\mathcal{H}$ such that an open neighborhood of $\partial \mathcal{H}$ lies on $S_{1} \cup S_{2}$, as desired. Further, choosing $\delta$ sufficiently small, we can make sure that the graph of $f$ is within a distance $\epsilon$ of $[-a, a]$ which in turn yields that $\mathcal{H}$ is within an $\epsilon$ neighborhood of $p q$.

We will construct $f$ by producing its graph. To this end, let $C_{1}$ be the intersections of $S_{1}$ with the $x y$-plane, and $\gamma(t), 0 \leq t \leq \ell$, be a unit speed parametrization for the portion of $C_{1}$ which lies above the $x$-axis and between the lines $x=-a-\delta$ and $x=-a-\delta / 2$. We assume that $\gamma(0)$ lies on the line $x=-a-\delta$. Let $\kappa:[0, \ell] \rightarrow \mathbf{R}$ be the signed curvature function of $\gamma$. Then $\kappa(p)=-1 / r$ (in our convention, the signed curvature is negative if the unit tangent vector to the curve is moving clockwise and is positive if it is moving counterclockwise). Let $\bar{\kappa}:[0, \ell+\eta] \rightarrow \mathbf{R}$ be a nondecreasing smooth extension of $\kappa$ such that $\bar{\kappa}$ is zero only at one point and $\bar{\kappa}(\ell+\eta)=1$ (one may easily construct $\bar{\kappa}$ using a step function). By the fundamental theorem of planar curves, $\bar{\kappa}$ determines a planar smooth curve $\bar{\gamma}$ with unit speed and curvature $\bar{\kappa}$ such that $\bar{\gamma}=\gamma$ on $[0, \ell]$. By construction $\bar{\gamma}$ has precisely one inflection point and total length $\ell+\eta$. The latter implies that, choosing $\eta$ sufficiently small, we can make sure that the image of $\bar{\gamma}$ lies in the second quadrant of the $x y$-plane. Further note that the amount by which the unit tangent vector to $\bar{\gamma}$ rotates depends on the integral of $\bar{\kappa}$, and $\int_{\ell}^{\ell+\eta} \bar{\kappa}(t) d t$ can be made as small as desired, by choosing $\eta$ sufficiently small. So the tangent lines of $\bar{\gamma}$ on $[\ell, \ell+\eta]$ can be made as close to the tangent line of $\bar{\gamma}$ at $t=\ell$ as desired.

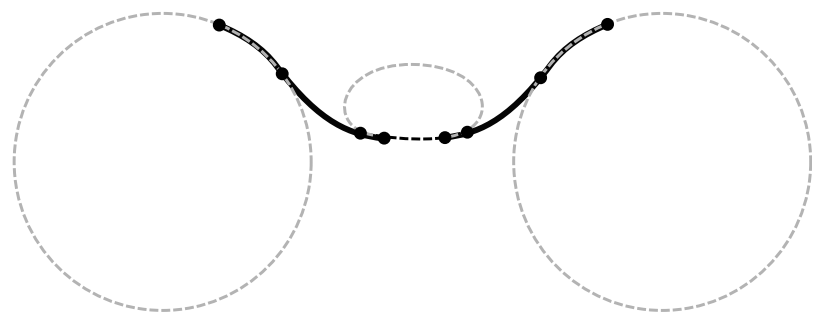

Figure 6

Now let $\bar{\gamma}_{1}:=\bar{\gamma}$, and $\bar{\gamma}_{2}$ be the reflection of $\bar{\gamma}$ across the $y$-axis; see Figure 6 . Then, by the last sentence in the above paragraph, if $\eta$ is small, we can make 
sure that $\bar{\gamma}_{2}[\ell, \ell+\eta]$ lies above all the tangent lines of $\bar{\gamma}_{1}[\ell, \ell+\eta]$ and vice versa. In particular, if we choose $\eta^{\prime}<\eta$ sufficiently close to $\eta$ so that $\bar{\kappa}$ is positive on $\left[\ell+\eta^{\prime}, \ell+\eta\right]$, then it follows that that $\Gamma:=\bar{\gamma}_{1}\left(\left[\ell+\eta^{\prime}, \ell+\eta\right]\right) \cup \bar{\gamma}_{2}\left(\left[\ell+\eta^{\prime}, \ell+\eta\right]\right)$ is strictly convex. So, by Lemma 3.3 , there exists a smooth simple closed curve $O$ of positive curvature such that $\Gamma \subset O$. Further, since $\Gamma$ lies above the $x$-axis, we may assume, by (the second sentence of) Lemma 3.3, that $O$ lies above the $x$ axis as well. Let $O_{1}$ be the bottom component of $O-\Gamma$. Then $\bar{\gamma}_{1}([0, \ell+\eta]) \cup O_{1} \cup \bar{\gamma}_{2}([0, \ell+\eta])$ is the graph of the desired function $f$.

Now we are ready to prove the main result of this section:

Proof of Proposition 3.1. Since $M$ has positive Gauss curvature at $p, q$ it follows that, given any open neighborhood $U$ of $\{p, q\}$ in $M$, we may let $V_{p}$, $V_{q} \subset U$ be open neighborhoods of $p$ and $q$ respectively such that $V_{p} \cap V_{q}=\emptyset$, $\bar{V}_{p}$ and $\bar{V}_{q}$ are strictly convex, and $\left.\nu\right|_{\bar{V}_{p}},\left.\nu\right|_{\bar{V}_{q}}$ are diffeomorphisms into geodesically convex subsets of $\mathbf{S}^{2}$, where $\nu$ is the Gauss map of $M$. By Lemma 3.4, we may deform $M$ inside $V_{p}$ and $V_{q}$, without changing the sign of curvature there or perturbing the tangent planes at $p, q$, so as to obtain a smooth embedded surface $M^{\prime}$, homeomorphic to $M$, which is spherical in neighborhoods of $p, q$, and contains $M-U$. Since (i) $M^{\prime}$ meets the end points of the segment $p q$ transversally, (ii) $M^{\prime}$ can be made arbitrarily close to $M$, and by assumption (iii) $M$ does not intersect the interior of the segment $p q$, it follows that we can make sure $M^{\prime}$ does not intersect the interior of $p q$. Thus $p, q$ face away from each other as points of $M$. Finally note that, by Lemma 3.6, $M^{\prime}$ has connected shades.

Now let $W_{p}, W_{q}$ be small open neighborhoods of $p, q$ in $M^{\prime}$ which are convex caps cut off from $M$ by planes parallel to $T_{p} M^{\prime}$ and $T_{q} M^{\prime}$ respectively. We may assume that $W_{p}$ and $W_{q}$ are so small that their closures lie inside the spherical open neighborhoods of $M^{\prime}$ at $p$ and $q$. Then, by Lemma 3.5, $M^{\prime \prime}:=M^{\prime}-\left(W_{p} \cup W_{q}\right)$ has connected shades. By Lemma 3.8 we may construct an hour glass $\mathcal{H}$, bounded by $\partial M^{\prime \prime}=\partial W_{p} \cup \partial W_{q}$, such that a neighborhood of $\partial \mathcal{H}$ coincides with a pair of collars of $\bar{W}_{p}$ and $\bar{W}_{q}$. Then, gluing $M^{\prime \prime}$ to $\mathcal{H}$ along their common boundary yields a smooth closed surface $\widetilde{M}$, which, by Lemma 3.2, has connected shades.

Next note that $\mathcal{H}$ lies within the convex hull of $W_{p} \cup W_{q}$. Thus, choosing $W_{p}, W_{q}$ sufficiently small, we can make sure that $\mathcal{H}$ is arbitrarily close to $p q$, which is disjoint from $M^{\prime \prime}$. So we can make sure that $\widetilde{M}$ is embedded. Finally, since $\widetilde{M}-M \subset\left(V_{p} \cup V_{q} \cup \mathcal{H}\right)$, choosing $V_{p}$ and $V_{q}$ sufficiently small, ensures that $\widetilde{M}-M$ is as close to $p q$ as desired. 


\section{Proof of Theorem 1.1}

We need only one more lemma. Let $\gamma: \mathbf{R} / \ell \rightarrow \mathbf{R}^{3}$ be a smooth simple loop, and $\operatorname{dist}_{\gamma}: \mathbf{R}^{3} \rightarrow \mathbf{R}$, given by

$$
\operatorname{dist}_{\gamma}(p):=\inf \{\|p-\gamma(t)\| \mid t \in \mathbf{R} / \ell\},
$$

be the distance function of $\gamma$. Then it follows from the tubular neighborhood theorem that for sufficiently small $r$

$$
\operatorname{Tube}_{r}(\gamma):=\operatorname{dist}_{\gamma}^{-1}(r),
$$

is a smooth embedded surface.

Lemma 4.1. Let $\gamma: \mathbf{R} / \ell \rightarrow \mathbf{R}^{3}$ be a smooth simple loop with nonvanishing curvature, $r$ be sufficiently small so that $M:=\operatorname{Tube}_{r}(\gamma)$ is a smooth embedded surface, and $K$ be the Gauss curvature of $M$. Then for every $t \in \mathbf{R} / \ell$,

$$
K(\gamma(t)-r N(t))>0
$$

where $N$ is the principal normal of $\gamma$.

Proof. Let $B:=T \times N$ denote the binormal of $\gamma$. Then $M$ may be parametrized by $X: \mathbf{R} / \ell \times \mathbf{R} / 2 \pi \rightarrow \mathbf{R}^{3}$ given by

$$
X(t, \theta):=\gamma(t)+r \cos (\theta) N(t)+r \sin (\theta) B(t) .
$$

A standard computation, with the aid of Frenet-Serret formulas, then shows that

$$
K(X(t, \theta))=\frac{-\kappa(t) \cos (\theta)+r\left(\cos ^{2}(\theta) \kappa^{2}(t)-\sin ^{2}(\theta) \tau^{2}(t)\right)}{r(1-r \cos (\theta) \kappa(t))^{2}},
$$

where $\kappa$ and $\tau$ are the curvature and torsion of $\gamma$ respectively. In particular, note that $K(\gamma(t)-r N(t))=K(X(t,-\pi))>0$.

Now we are ready to prove Theorem 1.1. First recall that the case of $g(M)=$ 0 is trivial, since the standard sphere $\mathbf{S}^{2}$ has connected shades, and the case of $g(M)=1$ was proved in [5], by showing that a tube around a skew loop has connected shades. For the case of $g(M)=n \geq 2$, let

$$
p_{i}:=(0,0, i), \quad \text { and } \quad N_{i}:=\left(0,0,(-1)^{i}\right),
$$

for $i=1, \ldots, 2 n-2$. Then, by Proposition 2.1, there exists a smooth simple skew loop $\gamma: \mathbf{R} /(2 n-2) \rightarrow \mathbf{R}^{3}$ such that $\gamma(i)=p_{i}$ and $N(i)=N_{i}$ (to apply Proposition 2.1, we only need to note that one may easily find $T_{i} \in \mathbf{S}^{2}$ such that $\left\langle T_{i}, N_{i}\right\rangle=0$, and $T_{i} \neq \pm T_{j}$, whenever $i \neq j$; for instance, let $\left.T_{i}:=(\cos (\pi i / 4 n), \sin (\pi i / 4 n), 0)\right)$. By Lemma 2.4, after a perturbation of $\gamma$, which keeps $\gamma$ fixed on an open neighborhood of each integer, we may assume that the $z$-axis does not intersect the image of $\gamma$ at any points other than $p_{i}$. 
Since $\gamma$ is a smooth simple skew loop, $M:=\operatorname{Tube}_{r}(\gamma)$ is a smoothly embedded surface with connected shades, for $r$ sufficiently small [5, Prop. 6.3]. Now, for $i=1, \ldots, n-1$, set

$$
x_{i}:=p_{2 i}-r N_{2 i}, \quad y_{i}:=p_{2 i-1}-r N_{2 i-1} ;
$$

see Figure 7. Then $x_{i}, y_{i} \in M$. We claim that $x_{i}, y_{i}$ 'face away' from each other,

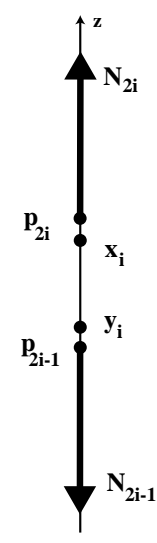

FiguRE 7

as defined in the beginning of Section 3. To see this first note that if $K$ denotes the Gauss curvature of $M$, then $K\left(x_{i}\right), K\left(y_{i}\right)>0$ by Lemma 4.1. Further, the segments $x_{i} y_{i}$ are pairwise disjoint from each other, and their interiors are disjoint from $M$, if we assume that $r$ is sufficiently small. Furthermore, if $\nu$ is the inward unit normal of $M$, then $\nu\left(x_{i}\right)=N_{2 i}$ and $\nu\left(y_{i}\right)=N_{2 i-1}$ (for, as a computation shows, if $X(t, \theta)$ is the parametrization of $M$ given by (4.1), then $\nu(X(t, \theta))=-\cos (\theta) N(t)-\sin (\theta) B(t))$. So the segment $x_{i} y_{i}$ is orthogonal to $M$ at its end points. Finally note that, since by definition $N_{2 i}$ is parallel to the positive direction of the $z$-axis, $p_{2 i}$ is a local minimum for the height of $\gamma$ (with respect to he $x y$-plane). This yields that $x_{i}$ is a local minimum for the height of $M$. Thus if $H$ is the mean curvature vector of $M$, then $H\left(x_{i}\right)$ points up, i.e., $H\left(x_{i}\right) \| x_{i}-y_{i}$. Similarly, since $N_{2 i-1}$ is parallel to the negative direction of the $z$-axis, it follows that $H\left(y_{i}\right) \| y_{i}-x_{i}$. Thus we conclude that $M$ has $n-1$ pairs of points $\left\{x_{i}, y_{i}\right\}$ which face away from each other.

Let $U_{i}$ be an open neighborhood of $\left\{x_{i}, y_{i}\right\}$ in $M$ chosen sufficiently small so that $U_{i} \cap U_{j}=\emptyset$, when $i \neq j$, and let $\epsilon>0$ be smaller than the smallest distance between the segments $x_{i} y_{i}$. By Proposition 3.1 there exists a smooth embedded surface $M_{1}$ with connected shades, and $g\left(M_{1}\right)=g(M)+1=2$, such that $M-U_{1} \subset M_{1}$ and $M_{1}-M$ lies within a distance $\epsilon$ of $x_{1} y_{1}$. In particular, $M_{1}$ has $n-2$ pairs of points $\left\{x_{i}, y_{i}\right\}, i=2, \ldots, n-1$, which face away from each other, and $M_{1}$ is disjoint from the interiors of $x_{i} y_{i}$. So we may 
apply Proposition 3.1 again. Repeating this procedure, we eventually obtain a smooth embedded surface $M_{n-1}$ with connected shades and $g\left(M_{n-1}\right)=n$.

\section{ACKNOWLEDGEMENT}

Parts of this work were completed while the author visited the Department of Mathematics at Pennsylvania State University.

\section{REFERENCES}

[1] T. F. Banchoff. The two-piece property and tight $n$-manifolds-with-boundary in $E^{n}$. Trans. Amer. Math. Soc., 161:259-267, 1971.

[2] T. E. Cecil and P. J. Ryan. Tight and taut immersions of manifolds. Pitman (Advanced Publishing Program), Boston, Mass., 1985.

[3] J. Choe. Index, vision number and stability of complete minimal surfaces. Arch. Rational Mech. Anal., 109(3):195-212, 1990.

[4] M. Ghomi. Solution to the shadow problem in 3-space. In Minimal Surfaces, Geometric Analysis and Symplectic Geometry, Advanced Studies in Pure Mathematics, vol. 29, 2000.

[5] M. Ghomi. Shadows and convexity of surfaces. Ann. of Math. (2), 155(1):281-293, 2002.

[6] M. Ghomi. Gauss map, topology, and convexity of hypersurfaces with nonvanishing curvature. Topology, 41(1):107-117, 2002.

[7] M. Ghomi. Strictly convex submanifolds and hypersurfaces of positive curvature. $J$. Differential Geom., 57:239-271, 2001.

[8] M. Ghomi. Nonexistence of skew loops on ellipsoids. Proc. Amer. Math. Soc, to appear.

[9] M. Ghomi. Tangent bundle embeddings of manifolds. Comment. Math. Helv. to appear.

[10] M. Ghomi and S. Tabachnikov. Totally skew embeddings of manifolds. Preprint.

[11] M. Ghomi and B. Solomon. Skew loops and quadric surfaces. Comment. Math. Helv., 77:767-782, 2002.

[12] A. Ben Hamza and Hamid Krim. A topological skeleton of illuminated manifolds. Proc. IEEE International Conference on Image Processing, Barcelona, Spain 2003.

[13] B. K. P. Horn and M. J. Brooks, editors. Shape from shading. MIT Press, Cambridge, MA, 1989.

[14] N. H. Kuiper. Geometry in curvature theory. In Tight and taut submanifolds (Berkeley, CA, 1994), pages 1-50. Cambridge Univ. Press, Cambridge, 1997.

[15] R. Schneider. Convex bodies: the Brunn-Minkowski theory. Cambridge University Press, Cambridge, 1993.

[16] B. Segre. Sulle coppie di tangenti fra ioro parallele relative ad una curve chuisa sghemba. Hommage au Professuer Lucien Godeaux, 141-167, Libraire Universitaire, Louvain, 1968.

[17] J. Sha and B. Solomon. No skew branes on non-degenerate hyperquadrics. Preprint.

[18] S. Tabachnikov and Y. Tyurina. Existence and non-existence of skew branes. Preprint.

[19] H. C. Wente. Personal email. January 9, 1999.

School of Mathematics, Georgia Institute of Technology, Atlanta, GA 30332

E-mail address: ghomi@math.gatech.edu

$U R L$ : www. math.gatech.edu/ ghomi 